Historic, archived document

Do not assume content reflects current scientific knowledge, policies, or practices. 

UNADILLA NURSERY GARDENS

UNADILLA, NEW YORK

\title{
FALL TRADE LIST
}

\author{
Wholesale and Surplus of
}

LANDSCAPE SPECIALTIES

\author{
Sept. 1, 1924
}

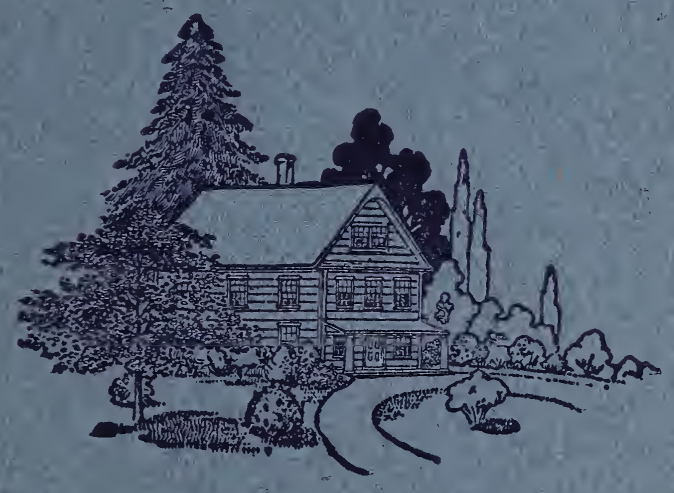

Our plants are grown for the Landscape trade, hardy, vigorous, cold resisting. Nursery 1000 feet above sea level.

Shipping facilities: By Truck, Freight car lots, Express, Parcels Post, the Delaware and Hudson Railway Company from Unadilla, the New York Ontario and Western Railway Company from Sidney. One day freight service to New York City. 


\section{$\because 78=x^{28}=10$}

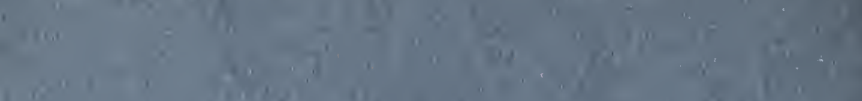

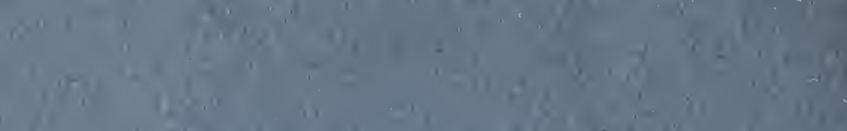

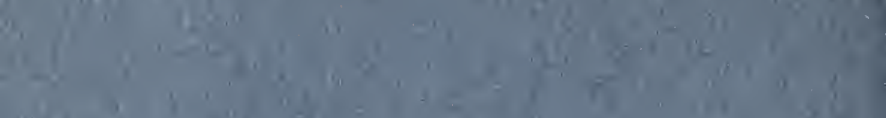

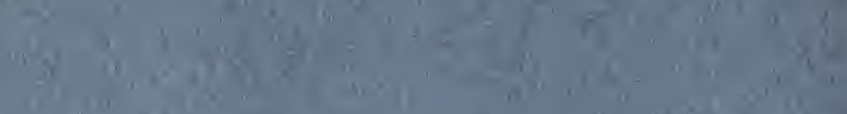

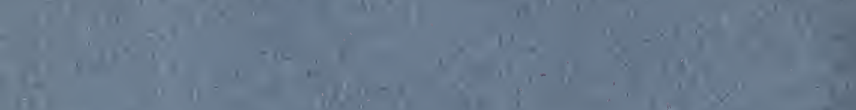

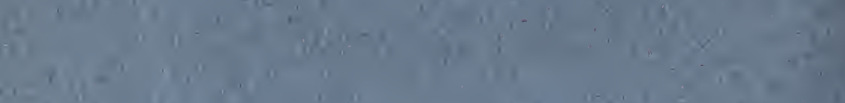

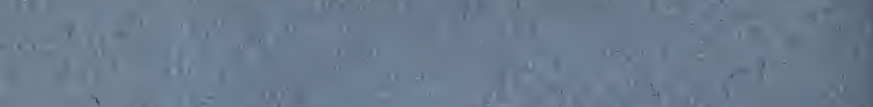

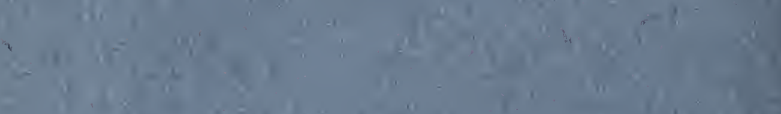
bex

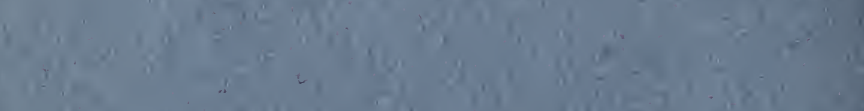

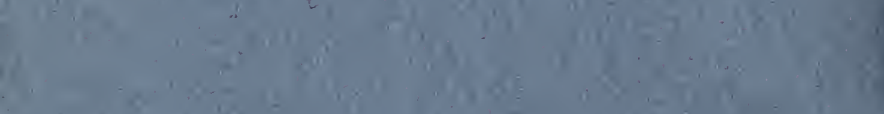

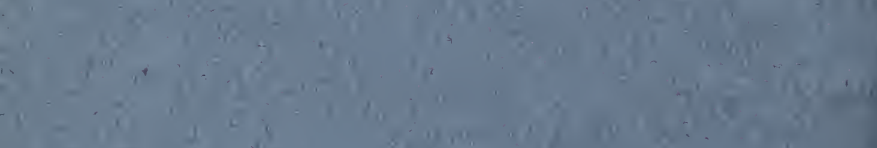

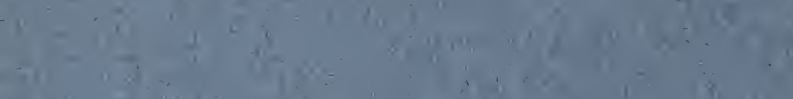

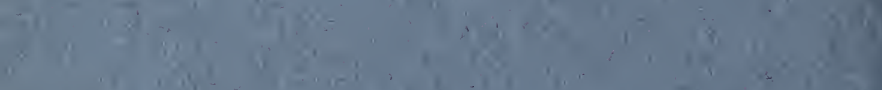

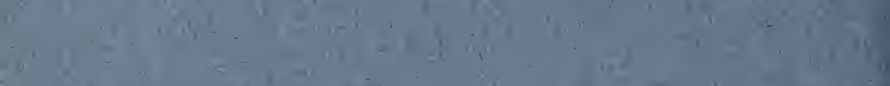

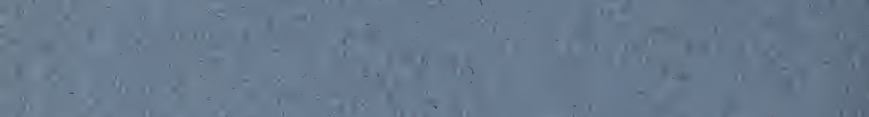

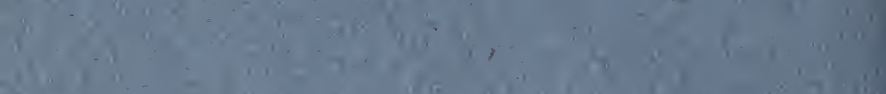

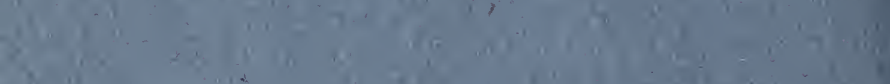

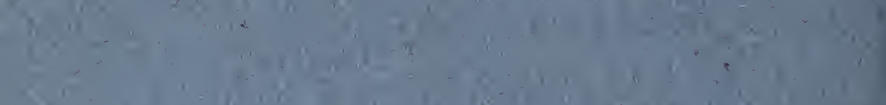

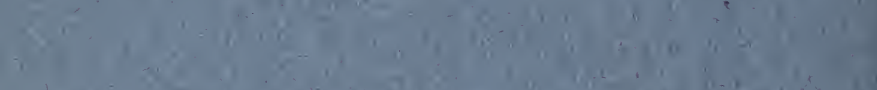

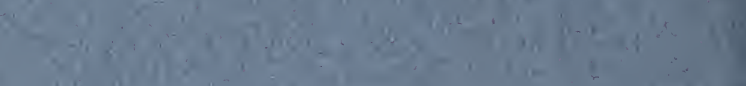

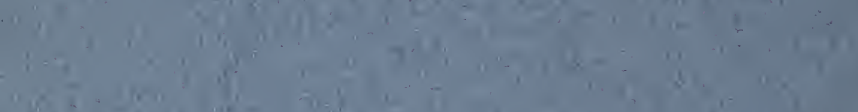

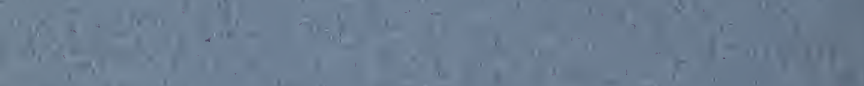

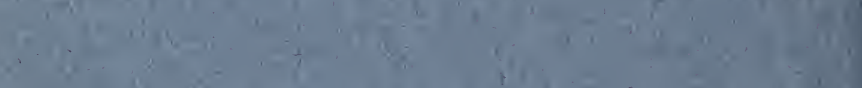

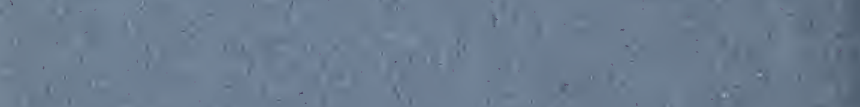

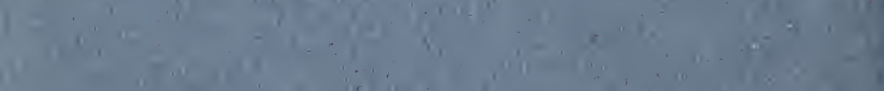

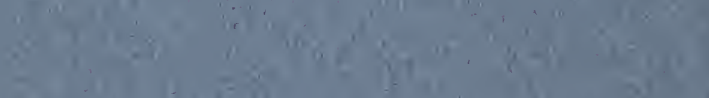

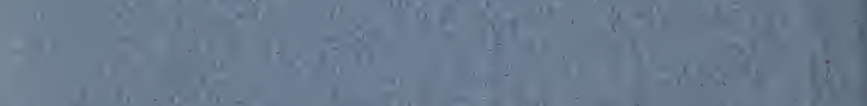

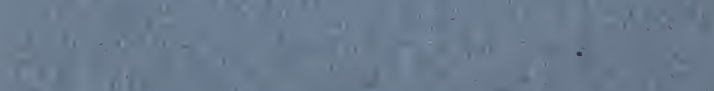

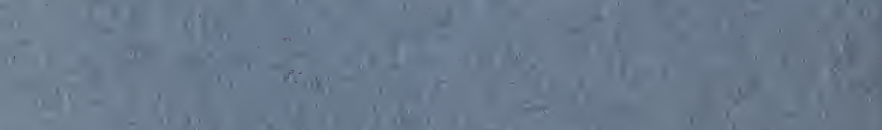
$=\left(\begin{array}{l}0 \\ 0\end{array}\right.$ 


\section{UNADILLA NURSERY GARDENS}

\section{UNADILLA, NEW YORK}

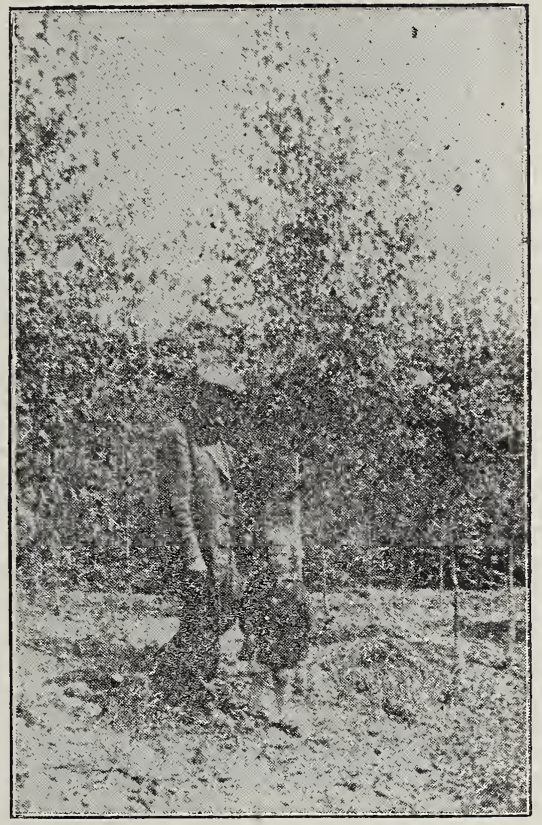

Specimens

(Cancells all previous Price Lists.)

BURLAPING CHARGES

Each

Small sizes

$10 \%$

2-3 ft. $15 \phi$

3-4 ft. $18 \phi$

4-5 ft. $21 \phi$

5-7 ft. $25 \phi$

7-9 ft. $35 \phi$

If trees in order are to be B. \& B. please include same when remitting.

Orders baled or boxed free, $5 \%$ discount, for cash with order.

Credit terms are net, boxing extra at cost. 


\section{LANDSCAPE EVERGREENS}

$\mathrm{X}$ means number of times transplanted.

Abies Balsum

Each

$2 \mathrm{xx} 18-24 \mathrm{in}$.

Balsum Fir

Abies Concolor

$6 \mathrm{xx} 2-3 \mathrm{ft}$.

Concolor Fir

Abies Fraserii

1.00

Fraser's Fir

$54 \mathrm{xxx} 6-7 \mathrm{ft}$.

6.00

$100 \mathrm{xxx} 8-9 \mathrm{ft}$.

10.00

Abies Douglassi, see Pseudtsuga Douglassi

Juniperus Communis Depressi

Common Juniperus Canadensis

$350 \times 10-15$ in.

$200 \mathrm{xxx} 12-18$ in. $\ldots \ldots \ldots$

$150 \mathrm{xxx} 18-24$ in.

Juniperus Horizontalis $10 \mathrm{xxx} 18-24 \mathrm{in}$.

Spreading Sabina

_............. 2.00

Picea Alba

White Spruce $10 \mathrm{xxx} 3-4 \mathrm{ft}$ 3.50

Picea Excelsa

$238 \times 10-12$ in.

Norway Spruce

$700 \mathrm{xx} 12-18$ in. $\ldots \ldots \ldots$

$600 \mathrm{xxx} 18-24$ in. $\ldots \ldots \ldots \ldots$

$50 \mathrm{xxx} 2-3 \mathrm{ft} . \ldots \ldots$

50 Trans. $3-4 \mathrm{ft}$.

100 Trans. 4-5 ft. . . . .

100 Trans. 5-6 ft. ............ 2.00

50 Trans. 6-7 ft.

Picea Pungens Colorado Blue Spruce

$25 \mathrm{xx} 8-12$ in. $\ldots . . .50$

$65 \mathrm{xx} 12-18$ in. $\ldots \ldots \ldots \ldots$

$13 \mathrm{xxx} 18 \mathrm{in.} \ldots \ldots$

200 xxx 18-24 in.

$162 \mathrm{xxx} 2 \mathrm{ft}$. Specimen

$127 \mathrm{xxx} 2-3 \mathrm{ft}$. Specimen Green -.- $\quad 3.00$

$262 \mathrm{xxx} 2-3 \mathrm{ft}$. Specimen Blue -..- $\quad 5.00$

15 xxx 3-4 ft. Specimen Green -..- 4.00

$14 \mathrm{xxx} 3-4 \mathrm{ft}$. Specimen Blue _...- 7.00

$1 \mathrm{xxx} 4-5 \mathrm{ft}$. Specimen Blue .... 9.00

Pinus Austriaca Austrian Pine

$1082 \mathrm{xx} 8-12$ in.

$300 \mathrm{xxx} 12-18$ in.

$300 \mathrm{xxx} \cdot 12-18$ in.

$50 \mathrm{xxx} 2-3 \mathrm{ft} . \ldots \ldots$

$20 \mathrm{xxx} 3-4 \mathrm{ft}$. $\ldots \ldots \ldots$

$6 \mathrm{xxx} 4-5 \mathrm{ft}$.

Pinus Banksania Jack Pine

500 xx $2-3 \mathrm{ft}$.

$20 \mathrm{xxx} 3-4 \mathrm{ft}$.

$10 \mathrm{xxx} 4-5 \mathrm{ft}$.

$10 \mathrm{xxx} 5-6 \mathrm{ft} . \ldots \ldots$

Pinus Mugho Dwarf Mountain Pine $50 \mathrm{xx} 6-8$ in.

$214 \mathrm{xxx} 18-24$ in. $\ldots . .00$

$100 \mathrm{xxx} 2-3 \mathrm{ft} . \ldots \ldots \ldots$ 
Pinus Resinosa

28 xx 18-24 in.

$25 \mathrm{xxx} 2-3 \mathrm{ft}$.

$10 \mathrm{xxx} 3-4 \mathrm{ft}$.

$7 \mathrm{xxx} 4-5 \mathrm{ft} . \ldots \ldots \ldots$

Pinus Sylvestris

Scotch Pine

Per 100

$100 \times 12-18$ in. ............ $\$ 20.00$

1060 xx 18-24 in. ............ 30.00

Pseudotsuga Douglassi

Douglas Spruce

Each Per 10 Per 100

620 xx 6-9 in. $\ldots \$ .15 \quad \$ 1.25 \$ \$ 120.00$

700 xxx 8-12 in. - $\quad .25 \quad 2.40 \quad 225.00$

$500 \mathrm{xxx} 12-18$ in. - $.40 \quad 3.50 \quad 325.00$

$50 \mathrm{xxx} 18-24$ in. $-.75 \quad 7.00$

$100 \mathrm{xxx} 2-3 \mathrm{ft}$. -.1 .50$

$84 \mathrm{xxxx} 3 \mathrm{ft} . \ldots-2.50$

$60 \operatorname{xxxx} 3-4 \mathrm{ft}$. ---3.00

Each

Retinospora Filifera Thread Branched Cypress 12 xxxx 2 ft. ................ 2.00

Retinospora Plumosa Aurea, Golden Plumed Cypress $25 \mathrm{xxx} 6-8$ in.

$100 \mathrm{xxx} 12-18 \mathrm{in.} \ldots \ldots$

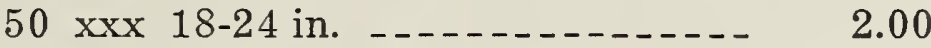

Retinospora Plumosa Green Green

$35 \mathrm{xxx} 12-18$ in.

Retinospora Squarrosa Veitchii Silver Cypress $100 \mathrm{xxx} 12-18$ in. ............. 1.25

\section{Thuya Occidentalis Per 100}

Per 100

$1000 \mathrm{xx} 12-15 \mathrm{in.} \ldots \ldots .00$

Each

$400 \mathrm{xx} 18-24$ in. .............. .50

125 xx $2-3 \mathrm{ft}$.

$40 \mathrm{xx} 3 \mathrm{ft} . \ldots \ldots$

25 x 4-5 ft. -

$25 \times 5-6 \mathrm{ft} . \ldots 3.00$

20 × 6-8 ft. $\ldots \ldots$

$50 \quad 8-10 \mathrm{ft}$. Specimen _....... $\quad 5.00$

$50 \quad 10-12 \mathrm{ft}$. Specimen

Thuya Occidentalis Compacta Compact Arborvitae

$50 \mathrm{xx} 12-18$ in.

50 xx 18-24 in.

$1 \mathrm{xxx} 30$ in.

Thuya Occidentalis Globosa Globe Arborvitae

$6 \mathrm{xxx} 18$ in.

Thuya Occidentalis Hoveyii Hovey's Arborvitae

Per 100

$600 \mathrm{xx}$ 9-12 in.

Each

$30 \mathrm{xx} 12-15$ in.

Thuya Oc. Pyramidal

Pyramidal Arborvitae

$250 \mathrm{xx}$ 18-24 in.

$250 \mathrm{xx} 2 \mathrm{ft.} \ldots \ldots \ldots$

$25 \mathrm{xx} 2-3 \mathrm{ft} . \ldots 200$ 


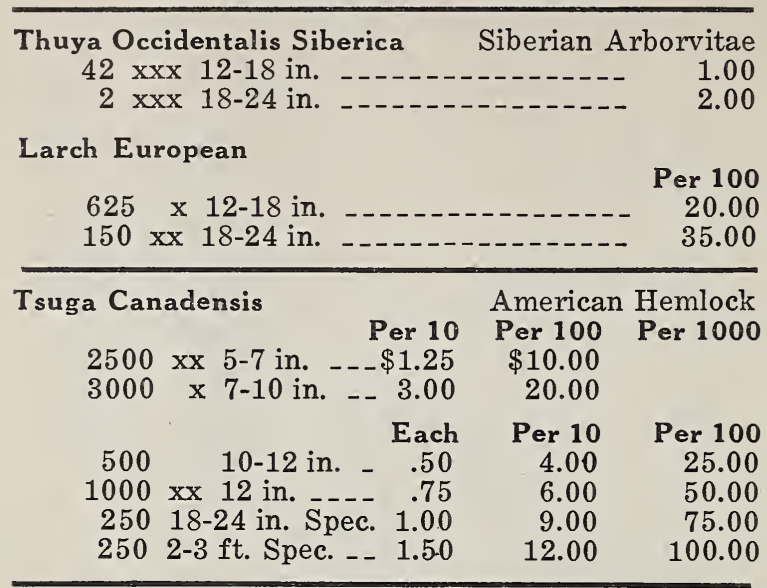

\section{EVERGREENS ONCE TRANSPLANTED}

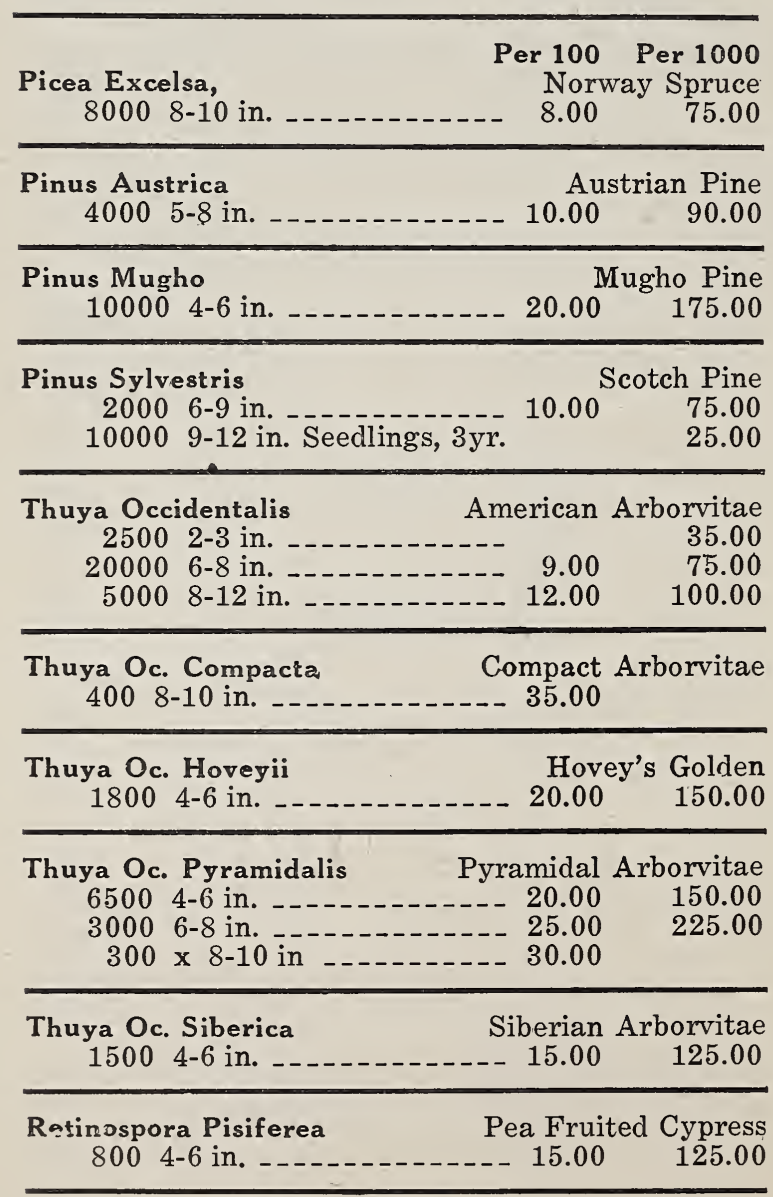


Retinospora Plumosa

Plumed Cypress

800 4-6 in.

16.00

130.00

Retinospora Plumosa Aurea, Golden Plumed Cypress 800 4-6 in.

20.00

175.00

Taxus Canadensis

American Yew $10006-8$ in. 15.00

125.00

\section{SHADE TREES}

Ash, American White

7-8 ft.

Each

8-10 ft.

10-12 ft.

12-15 ft.

$15-18 \mathrm{ft}$.

.50

1.00

Elm, American White

3-5 ft. -

$5-6 \mathrm{ft}$.

$8-10 \mathrm{ft} ., 11 / 4-11 / 2$ in.

$10-12 \mathrm{ft} ., 1 \frac{1}{2}-2$ in.

$2-2 \frac{1}{2}$ in.

$21 / 2-3$ in.

$3-4$ in.

NOTE-Special price quoted on elms in carload lots.

10-12 ft., 2-2 $1 / 2$ in.

Linden, American

Linden, European

1-2 ft. Transplants _...... . .08

$10-12 \mathrm{ft} ., 2-2 \frac{1}{2}$ in.

$12-15$ ft., $21 \frac{1}{2}-3$ in.

$14-15$ ft., 3 in.

Maple, Norway

$11 / 4-1 \frac{1}{2}$ in.

$11 / 2-13 / 4$ in.

$13 / 4-2$ in.

2.75

3.00

$2-21 / 2$ in.

4.00

$2-1 / 2-3$ in

$3-4$ in.

5.00

Note: Offering from this block of Norway Maples planted out twelve years ago, root pruned twice in trees from 2-4 in. caliper @ \$1.00 per inch in carload lots, gross diameter $1 \mathrm{ft}$. above the ground.

Special price on Elms and Maples in assorted sizes in carload lots.

Maple, Silver Pyramidalis

8-10 ft.

2.00

Maple, Silver

3-4 ft:

.25

5-6 ft.

.35

10-12 ft.

2.00

$20-25 \mathrm{ft}$., 3 in. diameter 
Maple, Sugar

$\begin{array}{lr}2-3 \mathrm{ft} . & .10 \\ 5-6 \mathrm{ft} . & .50 \\ 6-8 \mathrm{ft} . & \\ 8-10 \mathrm{ft} . & -\mathrm{r}\end{array}$

Maple, Red or Scarlet

4-7 in. Transplants _... .05

$2-3 \mathrm{ft}$.

$10-12 \mathrm{ft} ., 21 / 2-3$ in.

Maple Schwedlerii

$8-10 \mathrm{ft}$.

$12-15 \mathrm{ft}$.

Oak Pin

2-3 ft. - -

$5-6 \mathrm{ft}$.

$7-8 \mathrm{ft}$.

8-10 ft. -

$10-12 \mathrm{ft} ., 21 / 2$ in. caliper.

Oak Scarlet,

$5-6 \mathrm{ft}$.

Oak, Red

$5-6 \mathrm{ft}$.

$7-8 \mathrm{ft}$.

$10-12 \mathrm{ft}$.

$12-15 \mathrm{ft}$.

$15-20 \mathrm{ft}$.

Oak, White

6-7 ft. -

Walnut, or Hickory Shell Bark

$7-10 \mathrm{ft}$.

Walnut, Butternut or White Walnut

18-24 in. -

2-3 ft. -

3-5 ft.

.05

.10

.25

Walnut, Black

$12-18$ in.

10-12 ft.

.05

2.50

Walnut, Japan

18-24 in. .....

$6-8 \mathrm{ft}$.

$10-12 \mathrm{ft}$.

.05

.50

1.00

Willow Babylonica

8-10 ft.

Willow, Golden Weeping

$2-3 \mathrm{ft}$.

$3-4 \mathrm{ft}$.

$5-6 \mathrm{ft} . \mathrm{-}$

Willow, Laurel Leaf Weeping

$2-3 \mathrm{ft}$.

$3-4 \mathrm{ft}$.

Willow, Wisconsin Weeping

$4-5 \mathrm{ft}$.

$5-6 \mathrm{ft}$. 
Fringe, White

$2-3 \mathrm{ft}$.

Horsechestnut, White Flowering

4-5 $\mathrm{ft}$.

.50

$7-8 \mathrm{ft}$.

1.00

Hydrangea Tree

$2-3$ ft.

$3-4$ ft..- .90

4-5 ft.

Larch European

$12-18$ in.

18-24 in. -

$7-8 \mathrm{ft}$.

Prunus Pissardi

$3-4$ ft. -1.0 .75$

Rhus Typhine

3-5 ft.

Sassafras

3-4 $\mathrm{ft}$.

.25

4-5 ft.

.35

\section{SHRUBS}

Althea Amplissima, Double Red

Per 10 Per 100

18-24 in.

$1.00 \quad 8.00$

Althea Ardens, Double Purple

2-3 ft.

1.40

12.00

Althea Boule De Fue, Double Red 18-24 in. -..... 1.00

8.00

Althea Elegantissima, Pink 18-24 in.

$1.00 \quad 8.00$

Althea Joan of Arc

18-24 in.

1.00

8.00

Althea Peoniflora, Double Cherry Red 18-24 in.

1.00

8.00

Barberry Thunbergii 18-24 in.

2.75

25.00

Cornus Baileyii

2-3 $\mathrm{ft}$.

3.00

25.00

Cornus Elegantissima

2-3 ft.

4.50

40.00

\section{Cornus Florida}

18-24 in.

1.00

Cornus Sanguinea, Red Osier

$2-3 \mathrm{ft}$.

1.50

4-5 ft.

3.00

12.00

25.00

Cornus Siberica

1-2 ft.

1.20

10.00

Deutziá Crenata, Single White

18-24 in.

1.00

1.50

2-3 ft.

-

12.00 
Deutzia Gracilis,

$12-18$ in

Forsythia Intermedia, Golden Bell 18-24 in. .......... 1.50

Forsythia Suspensa, Drooping Golden 18-24 in. ...... 1.60

Forsythia Veridissima, Green-twigged $2-3 \mathrm{ft}$.

Hydrangea Aborescens, Hills of Snow

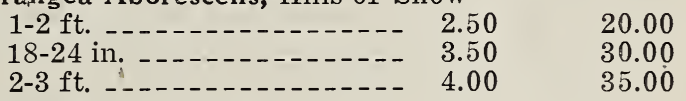

Hydrangea, P. G. Bush

$2 \mathrm{ft}$.

$4.00 \quad 35.00$

Hydrangea Tree

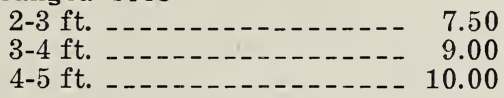

Lilac Purple

$1-2$ ft. $\ldots \ldots \ldots$.

$2-3$ ft. $-\ldots \ldots \ldots 00$

$3-4$ ft. $-\ldots-1.50 \quad 30.00$

$4-5$ ft.

Lilac White

$2-3$ ft. - $-1-28.00$

$3-4$ ft. $\ldots \ldots \ldots-\ldots 00$

Ligistrum Amurense, Amur River Privet North
$12-18$ in.
.90
18-24 in.
8.00
$2-3 \mathrm{ft}$.
1.25
10.00
$2.00 \quad 18.00$

Ligistrum Ibota, Ibota Privet

$\begin{array}{rrr}12-18 \text { in. } & & \\ 18-24 \text { in. }-1.00 \\ 2-3 \mathrm{ft} . \\ \end{array}$

$2-3 \mathrm{ft}$.

Ligistrum Ibolium, 6-12 br.
$2-3 \mathrm{ft}$.
2.50
18.00

$3 \mathrm{ft}$.

3.50

28.00

Ligistrum Regeliamum, Regal's Privet 18-24 in. -

20.00

Ligistrum Japonicum, Jap Privet 18-24 in.

Ligistrum Ovalifolium, Calif. Privet

$18-24$ in. ............ 2.50

$2-3 \mathrm{ft}$.

5.00

Ligistrum Polishii, Polish Privet

$12-18$ in

Lonicera Bella Alba

$\begin{array}{lll}2-3 \mathrm{ft} . & & \\ 4-5 \mathrm{ft} . & & \end{array}$

Lonicera Fra,grantissima, Fragrant

18-24 in. 
Lonicera Morrowii

$2-3 \mathrm{ft}$.

Lonicera Japonica, Japan Honeysuckle

$18-24$ in.

Lonicera Tartarica Alba

4-5 ft.

Philadelphus Coronarius Aurea

18-24 in.

$4.50 \quad 40.00$

Philadelphus Grandiflora, Mock Orange

$2-3 \mathrm{ft}$. .

$3-4 \mathrm{ft}$.

25.00

28.00

Rosa Rugosa, Hardy Jap Rose

$10-15$ in. -

$18-24$ in.

Sambucus Acutiloba, Cut Leaf Elder

$2-3 \mathrm{ft}$.

6-7 ft.

Sambucus Canadensis

$1 \mathrm{ft}$.

Sambucus Aurea, Golden Elder $2-3 \mathrm{ft}$.

Spirea Anthony Waterer $18-24$ in. _. $4.00 \quad 35.00$

Spirea Aurea

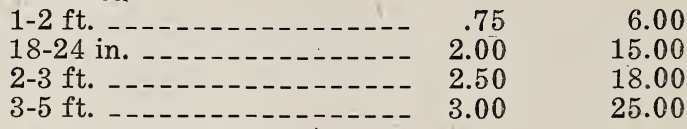

Spirea Tormentosa

$18-24$ in. -... $50 \quad 4.50$

Spirea Thunbergii

$2 \mathrm{ft.}$

Spirea Van Houttei

$18-24$ in.

$2-3$ ft.

$3-4$ ft.

Syringa Villosa

$2-3 \mathrm{ft.}$

Symphoricarpus Racemosus, Snowberry

18-24 in. . . .

$3-4$ ft.

Symphoricarpus Vulgaris, Coralberry

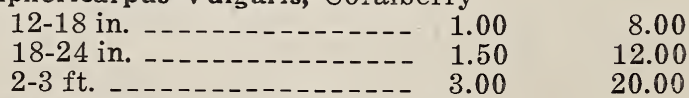

Wiegelia Rosea
$2-3 \mathrm{ft}$.
$3.00 \quad 25.00$

$3-4$ ft.

Wiegelia Variegated

18-24 in. -...... $4.00 \quad 35.00$

All shrubs nursery grown and one or more times transplanted. Many of these shrubs are especially desirable for the landscape trade. 


\section{VINES}

Ampelopsis Veitcheii, Boston Ivy

Per 100

18-24 in., 2 yr.

25.00

Celastus Scandens, Bitter Sweet

18-24 in., 2 yr.

20.00

Clematis Painiculata

Transplants, 2 yr., No. 1

20.00

Clematis Virgiana

18-24 in.

20.00

Euonymus Radicans Vegetus

12-18 in.

40.00

Euonymus Radicans, Variegated 12-18 in., 2 yr.

25.00

Lonicera Coral

$2 \mathrm{yr}$.

Lonicera, Hall's

$2 \mathrm{yr}$.

Pachysandra Terminalis, Japan Spurge

12-18 in., 2 yr.

25.00

Trumpet Vines

$3 \mathrm{yr}$.

20.00

Wistaria, Purple

Transplants, 4 yr.

30.00

Wistaria, White

Transplants, 3 yr.

40.00

Wistaria, Blue

Transplants, 3 yr.

40.00

\section{ROSES}

2yr. Field Budded, $\$ 35.00$ per 100

American Beauty

Red

A. R. Waddell _... Copper Pink

Betty -

Columbia

C. F. Meyer -

Frau Karl Druschki _..._... White

Gen Mac Arthur .

Gruss An Teplitz

Golden Ophelia _........ Yellow

J. L. Mock _._._._._._. Silvery Rose Pink

K. A. Victaria -

Los Angeles .

M. B. Job _._. Yellow

Magna Charter

Mrs. J. Laink _.

Opelia _..._. Salmon Rose

Paul Neyron _._._. Rose Pink

Radiance - .

Soleil D'or _._.

Ulrich Brunner .............. Light Red 


\section{RAMBLERS}

\section{Year Transplants}

Each

American Beauty _-_._- 40

Aviator Bleriot _....... 40

Baby Rambler, Red _... $\quad .40$

Blue Rambler _._-_._._._._. 35

Crimson Rambler _..._............ $\quad .40$

Dr. Van Fleet

Dorothy Perkins _... .25

Excelsa _..._._._.

Flower of Fairfield

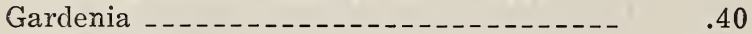

Paul's Scarlet _._._._._. 40

Seven Sisters _-_-_-_-_._- .25

Silver Moon

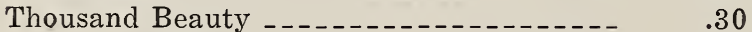

Yellow Rambler _._._._._._._. $\quad .35$

In buying ramblers it pays to buy good stocky plants.

\section{IRIS}

\section{$\$ 10.00$ per 100}

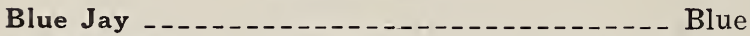

Honorabilis - Yellow Mme Cherreau ... White Bordered with Blue Pallida Speciosa Queen of May _..._._._. Soft Pink Mixed Varieties

\section{PEONIES}

Canary, Cream White

Delachei, Purple or Crimsom Violet.

Delicatissima, Rose Pink

Duc Des Enghien, Soft Red

Ducess De Nemours, Pure White

Duke of Wellington, Sulphur White

Festiva Maxima, Early White, Rose Type --

Felix Crousse, Flaming red _.........

Fideline, Violet Rose

Fragrans, Solferino Red _.....

Hercules, Shell Pink _-_._-_._.

Humeii, Cherry Pink ...........

La Tulipe, Lilac White

Louis Van Houttei, Dark Crimson

Magnifica, Cream White

Mme Chaumy, Cream White

Marechal Vaillant, Aniline Red -.--

Maude Hutchinson, Lilac White _......-

Princess Beatrice, Violet Rose _._._._-

Purple Queen, Purple

Queen Victoria, Flesh White

Whites

Pinks

Mixed
Per 100

30.00

32.00

32.00

30.00

35.00

30.00

30.00

40.00

30.00

30.00

25.00

20.00

35.00

40.00

30.00

35.00

35.00

25.00

30.00

35.00

30.00

25.00

22.00

18.00 


\section{PHLOX}

Strong Field Grown Plants, $\$ 12.00$ per 100

Australia - Claret Red Baron Van Dedem -..._._._. Crimson Scarlet Champs Elysees _._._._._-_. Rosy Magenta Coquelicot _. Eclaireur _........ Rosy Carmine, Light Halo Europa - - Ferdinand Cortez - . Frau Anton Buchner -.... White Shaded Pink La Vague . Lumineaux - . Mme. P. Dutrie - Lilac Rose Mrs. Jenkins _._. Best White

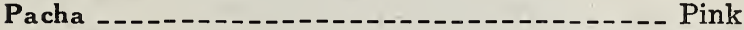
Pantheon -.......... Salmon Pink Rheinlander . . Salmon Pink Riverton Jewel _._._._._._._. Rose Rijnstrom - .

\section{GARDEN FRUITS}

Per 1000

Asparagus

Martha Washington

Rust Proof, 2 yr.

Per 100

\section{Currants}

Cherry, 3 yr.

Fay's, 3 yr.

Perfection, 3 yr. -

White Grape, 2 yr.

Wilder, 3 yr.

\section{Gooseberry}

Downing, 3 yr. _.

Houghton, 3 yr.

\section{Grapes}

Moore's Early, 2 yr., x

Niagara, 2 yr.

Worden, 2 yr. ..... $\quad 12.00$

Rhubarb

Hyatt's -

Sage

Holt's Mammoth _... 10.00

Strawberry Plants

Per 1000

Gandy

Dunlap -

Progressive

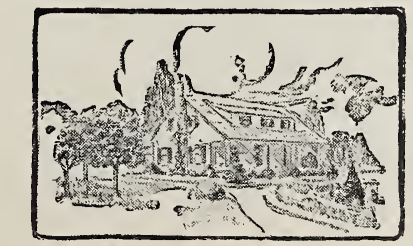




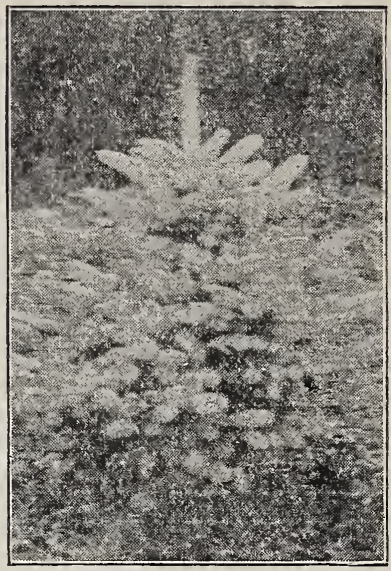

One of our Beautiful Blue Spruces.

If you want any of these trees get.busy.

\section{SPECIMEN EVERGREEN SHRUBS}

(Standard quality, five stalks of height stated)

Azalea Aborescens, Sweet Azalea

3-4 ft. Clumps _-...-

Andromeda Catesbaeii, Leucothoe Catesbaeii

One of the most graceful evergreen shrubs having dark green shiny foliage and gracefully arched branches, bell shaped white flowers early in May. The foliage turns bronze in fall. A very desirable shrub for underplanting with Rhododendrons and other large shrubs.

Clumps, 2-3 ft.

Azalea Calendulacea, Flame or Lutea

Beautiful, hardy, northern Azalea, color varies from yellow red to orange red to flame red and cream.

Clumps, 2-3 ft.

Each

Clumps, 3-4 $\mathrm{ft}$.

1.00

Azalea Nudiflora, Pinkster

1.50

Fragrant, rose pink blooms appearing with the unfolding of the leaves.

Clumps, 3-4 ft.

Daphne Mezerum, February Daphne

A most rare shrub having pinkish lilac like blooms in the early spring before the leaves appear.

Plants, $12-18$ in 
Kalmia Latafolia, Mountain Laurel

Native cut back

Specimen Clumps, 12-18 in

Specimen Clumps, 18-24 in. -

Specimen Clumps, 2-3 ft. -...- 1.25

Kalmia Latafolia, Mountain Laurel

Native, cut back clumps, grown in open fields.

Rhododendron Catawbiense, Rhododendron Catawba One of the most beautiful, hardy flowering shrubs having purple reddish flowers, thrives in the sun and shade even in the highest, coldest, peaks of the eastern mountain ranges.

x 2-3 ft. _..........................

Rhododendron Maximum, Great Laurel

This beautiful shrub bears white or light pink flowers in June or July. For plantings for natural effect along walks and drives, about foundations or in woody places there is nothing that produces more satisfaction than this shrub. Native cut back clumps.

Standard Clumps, 2-3 ft.

Each

Specimen Clumps, 2-3 ft. -

Extra Specimen Clumps, 2-3 ft. - - - - $\quad 3.50$

Standard Clumps, 3-4 ft. - - 3.00

Specimen Clumps, 3-4 ft.

Extra Specimen Clumps, 3-4 ft. - $\quad 5.00$

Standard Clumps, 4-5 ft. -

Specimen Clumps, 4-5 ft.

Super Specimen Clumps, $4-5 \mathrm{ft}$. - - - $\quad 6.00$

Standard Clumps, 5-6 ft. - - 4.00

Specimen Clumps, 5-6 ft. - .

Super Specimen Clumps, 5-6 ft. - - $\quad 8.00$

Vaccinium Corymbosum, High Bush Blueberry

One of the finest shrubs for border or evergreen planting. Leaves turn crimson and claret in autumn.

Fine Clumps, 18-24 in. -........ $\quad .50$

Stocky Plants, 2-3 ft.

Stocky Plants, 3-4 ft. - _._-_._- 1.00

\section{RHODODENDRON AND KALMIA PRICES, CAR LOTS}

Rhododendron Maximum, Great Laurel

Many firms are finding it advantageous to buy at least one car load shipped in the fall for the early spring rush. For the past two seasons it has been April fifteenth before we could move a plant from the collection field. This means that orders and landscape jobs are held up waiting for the plants to arrive. If you have never tried the fall shipments give it a trial this year, you will make good money on the early plants for season 1925. Our men have collected Rhododendrons for over twenty years. They know where the good plants are in the Catskill Mountains. 
Native, collected, and cut back clumps. These prices apply on solid car lots only. F. O. B. Car, our Rhododendron fields.
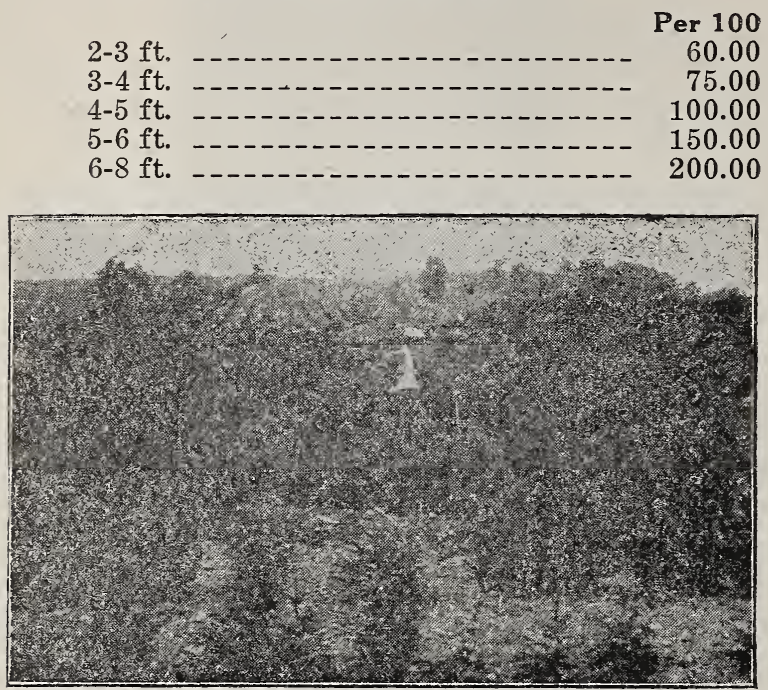

This shows a view of our nursery with evergreens in the foreground and a cherry orchard just back of the nursery.

$\begin{array}{cccc}\text { Plants per car single-deck } & \text { Double-deck cord-rooded } \\ \text { 1-2 ft. } & 400 & 600 & 1200 \\ 2-3 \mathrm{ft} . & 225 & 400 & 800 \\ 2-3 \mathrm{ft} . & 225 & 400 & 800 \\ 3-4 \mathrm{ft} . & 200 & 300 & 600 \\ 4-5 \mathrm{ft} . & 150 & & 500 \\ 5-6 \mathrm{ft} . & 100 & & 300 \\ 6-8 \mathrm{ft} . & 75 & & 150\end{array}$

$12-18$ in. Plants

Per 100

25.00

18-24 in.

2-3 ft.

50.00

Our plants are Upland Nursery Grown, from one to several times transplanted, producing plants of rare hardiness and vitality. These produce results when planted anywhere.

We can supply a long felt need in Ornamental Shrubs, Plants, and Trees, Rhododendrons, and Evergreens to meet your landscape plan in every detail. Send in your Want list with specifications without delay.

\section{Unadilla Nursery Gardens}

Member of

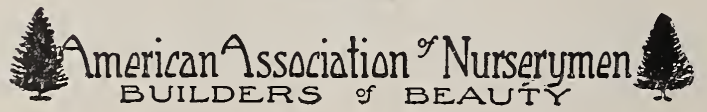





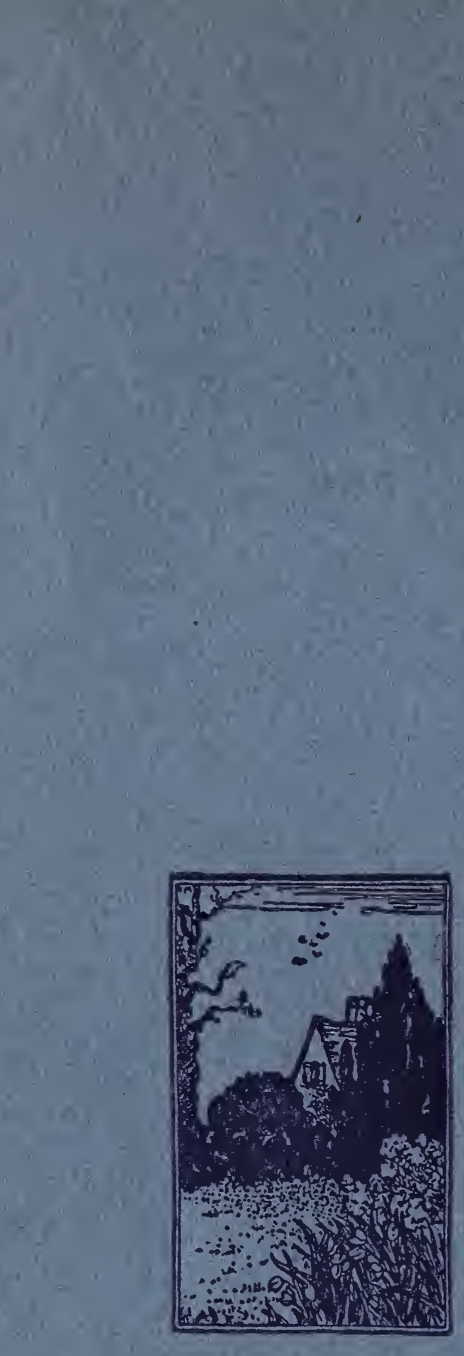

2

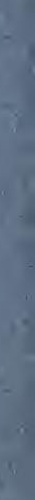

